\title{
HIGHLIGHTS
}

OBESITY

\section{Blocking serotonin receptor 3 prevents glucose-induced obesity in mice}

The increase in consumption of sweetened soft drinks, in particular, over the past 30 years, is associated with the prevalence of obesity in the USA. The intestinal serotonergic system is involved in sugar detection and the control of food intake, and Synia Weber and colleagues have now found that blocking serotonin receptor 3 (5-HT3R) in mice given glucose-sweetened drinking water prevents weight gain.

For 8 weeks mice were fed $30 \%$ glucose in their drinking water to make them obese, or given plain water as a control, in addition to their normal food. The researchers then studied the effect of the oral 5-HT3R antagonist tropisetron on body weight and caloric intake.

Treatment with tropisetron reduced intestinal motility and almost completely blocked weight gain in the mice that were fed glucose. "Surprisingly, the protective effect of tropisetron was neither accompanied by reduced caloric intake nor by decreased expression of the main gastrointestinal and liver glucose transporters," comments Stephan Bischoff, the corresponding author on the study. "Instead, we found that tropesitron normalized the increased expression of carbohydrate responsive element binding protein, fatty acid synthase and triglycerides in the liver of the mice fed on glucose," he adds. This suggests that tropisetron prevents the metabolism of glucose to trigylcerides in the liver.

In addition, mice fed on glucose and treated with tropisetron had increased expression of a protein that catalyzes the interconversion of the two major ketone bodies produced during fatty acid catabolism, and also increased levels of ketone bodies in the plasma. These data suggest that tropisetron may act by switching the metabolism of the mice

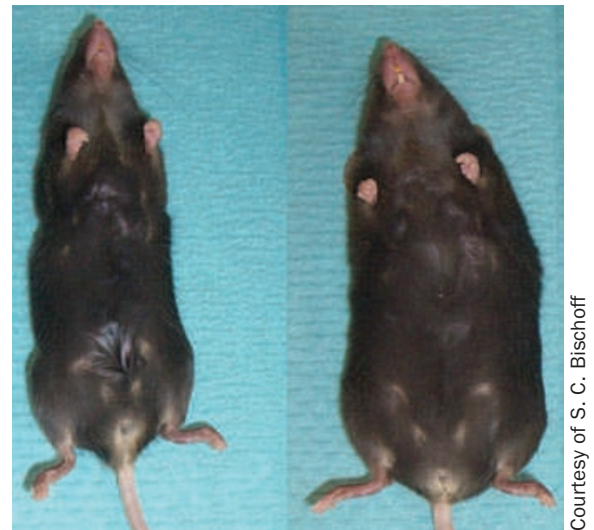

from an anabolic process, in which high glucose intake leads to the formation of triglycerides and weight gain, to a more catabolic metabolism.

These data indicate that 5-HT3R is a promising target for the modulation of hepatic glucose metabolism and for the prevention of obesity.

\section{Ezzie Hutchinson}

Original article Weber, S. et al. Treatment with the 5-HT3 antagonist tropisetron modulates glucose-induced obesity in mice. Int. J. Obes. 33, 1339-1347 (2009) 